\title{
Inhibiting more than the proton pump
}

\section{Dr Shaza Ahmed, Dr Chloe Broughton, Dr Beas Bhattacharya}

Great western Hospital Swindon

\section{Introduction}

- Hyponatraemia is defined as serum sodium concentration $<135 \mathrm{mmol} / \mathrm{L}$.

- It is the most common electrolyte disorder encountered in clinical practise Proton pump inhibitors (PPI's) are commonly prescribed in the UK, and the indication and duration of treatment is often not reviewed.

\section{Methods}

- A retrospective audit was performed of patients admitted to The Great Western Hospital (GWH) with a serum sodium of $127 \mathrm{mmol} / \mathrm{L}$ or less on admission, over a three month period.

- The aim was to identify prescribed medications that may be contributing to the hyponatraemia.

\section{Result}

Is this patient on drugs that might lead to hyponatraemia

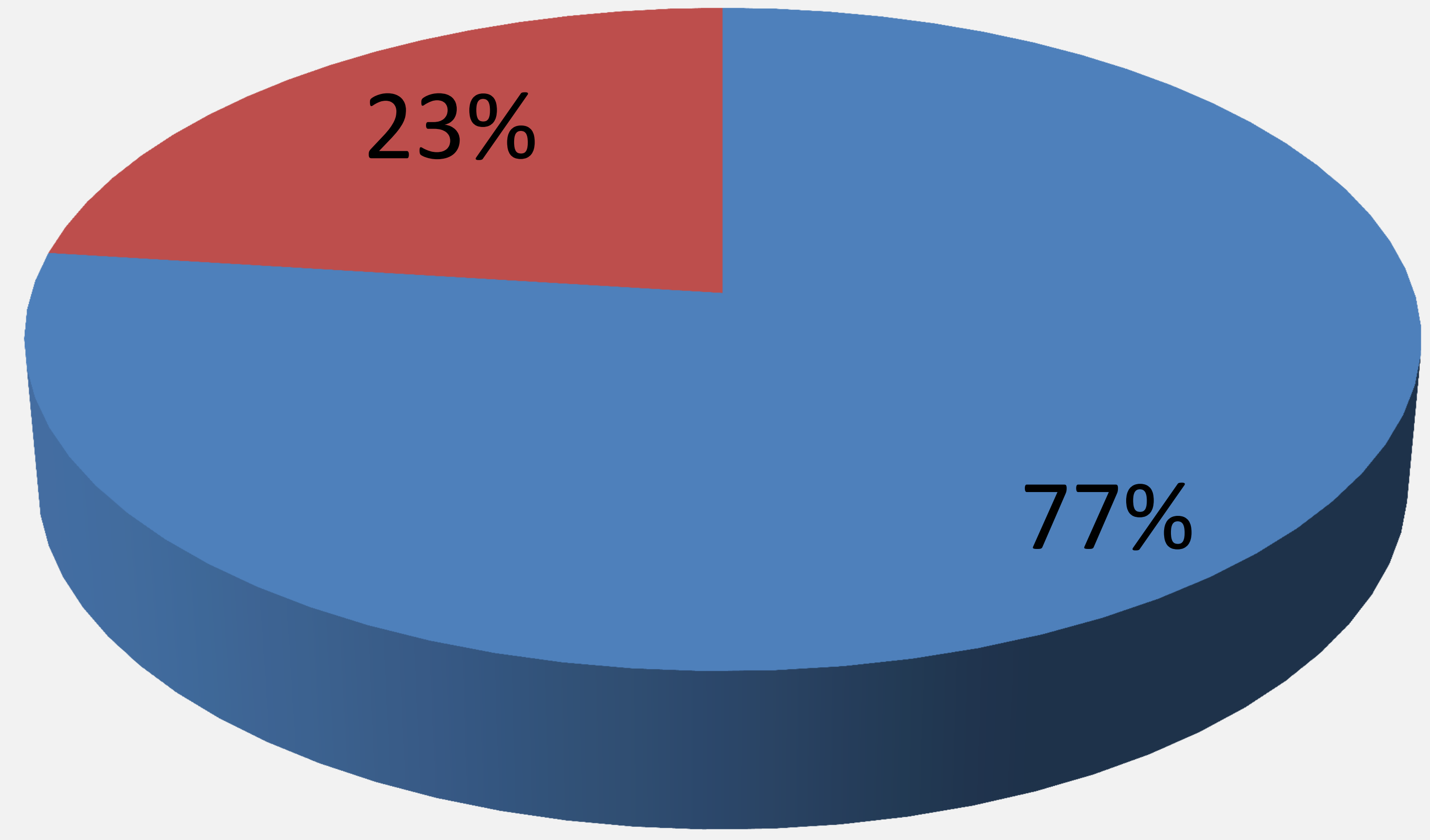

Does the patient have a previous diagnosis of hyponatraemia?

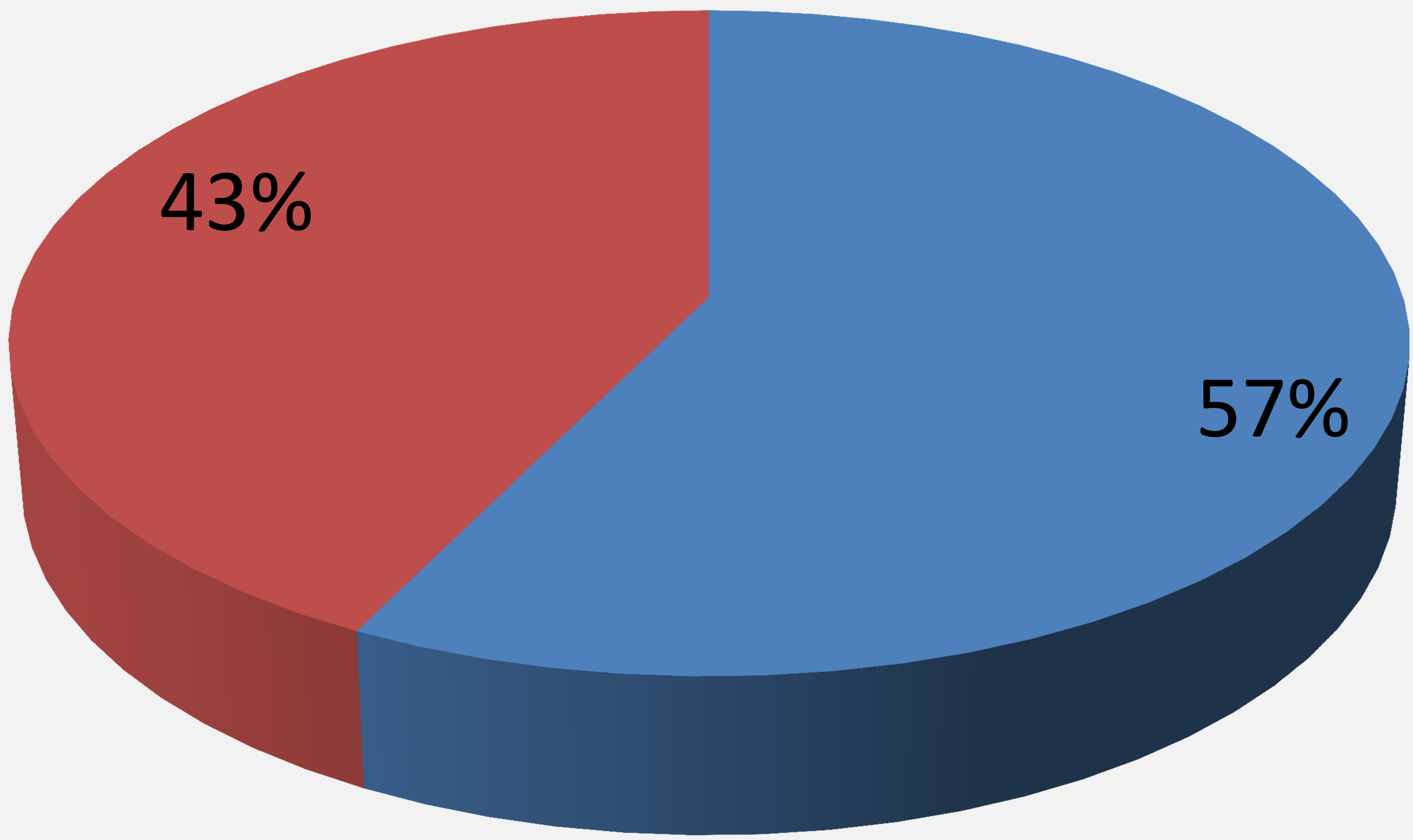

0

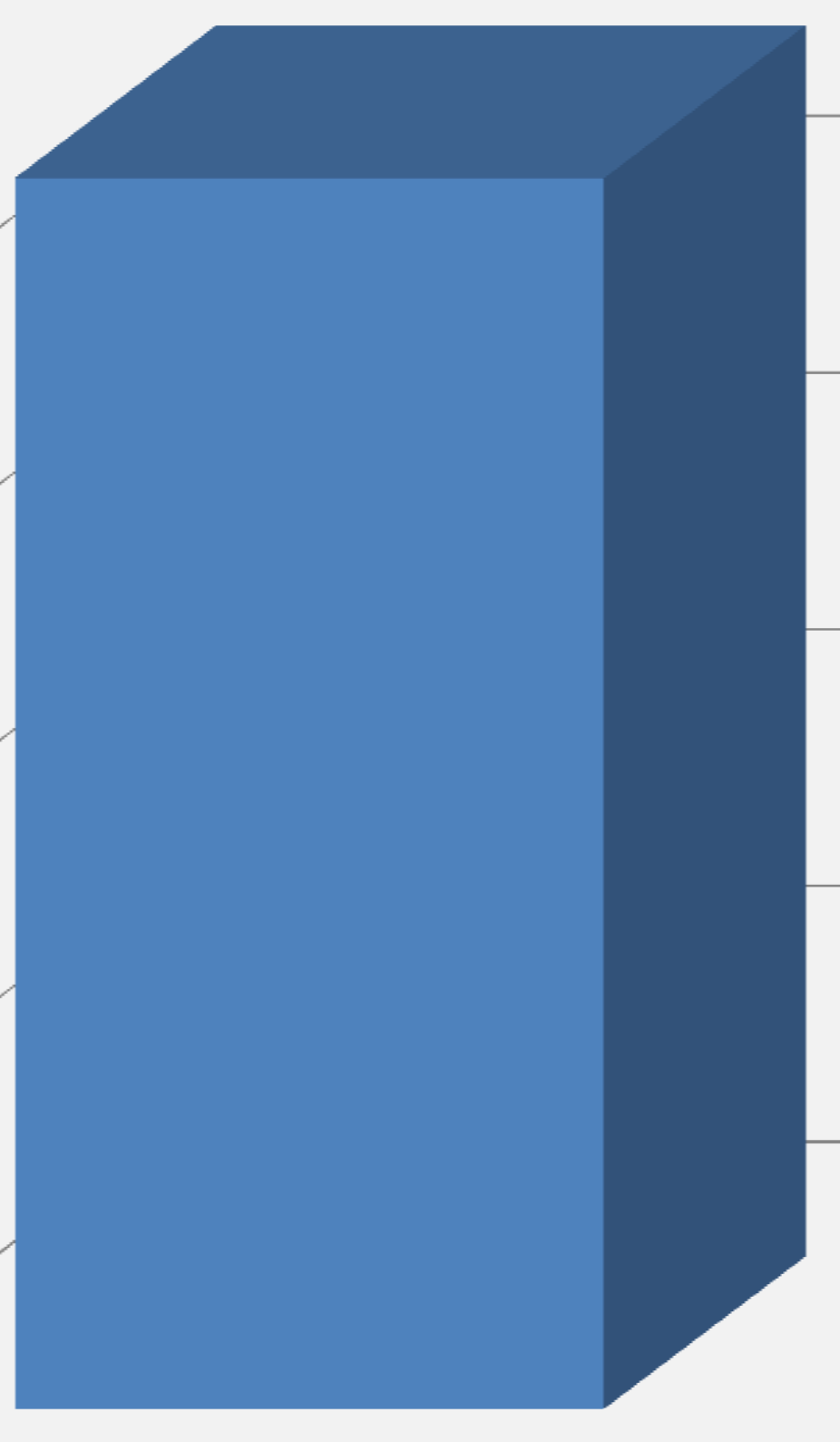

PPI

YES

NO

Diuretics

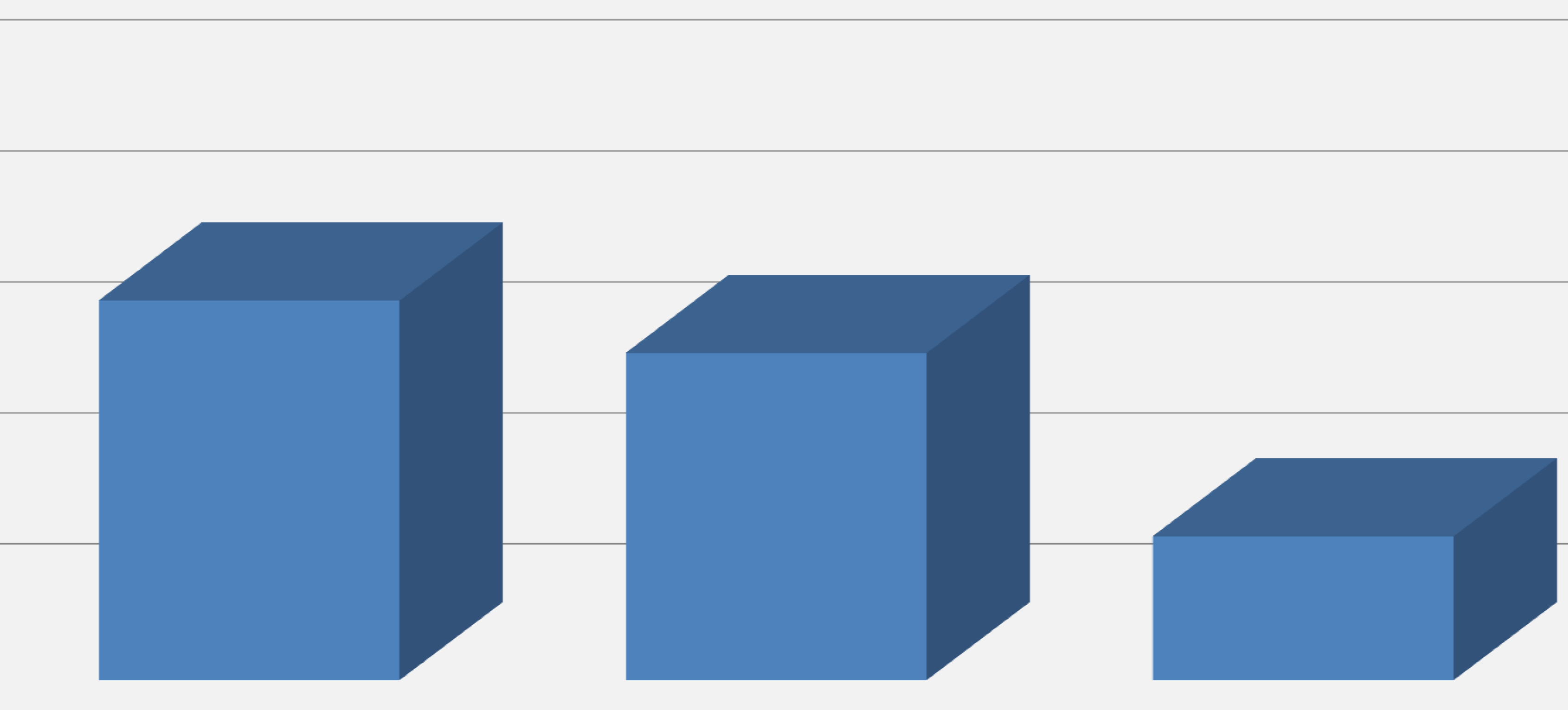

Antidepressants YES
Anti-epileptics

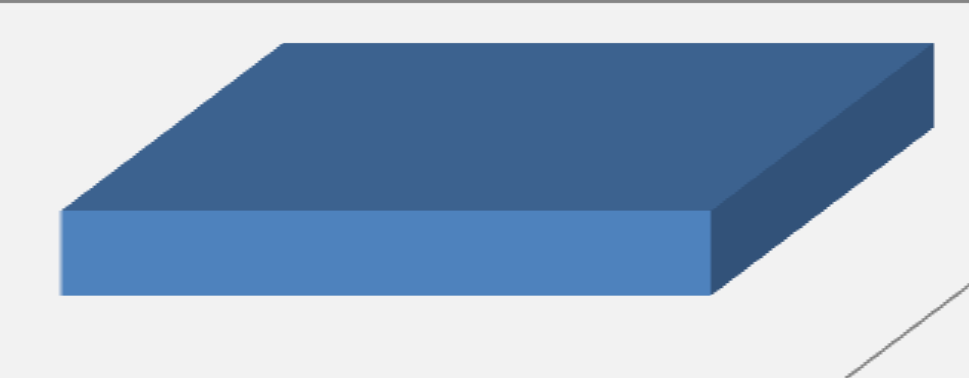

Others

\section{Discussion}

- Hyponatraemia is often caused by drugs such as Anti-psychotics, anti-depressants, diuretics

- This audit clearly demonstrates apart from the well known drugs, proton pump inhibitors is a significant contributor to hyponatraemia.

- There are often alternatives, for example $\mathrm{H} 2$ receptor antagonists rather than PPIs, and clinicians should consider these in patients at risk of hyponatraemia.

- $57 \%$ of patients audited had a previous diagnosis of hyponatraemia suggesting we may be missing opportunities to review prescribed medications 